\title{
Evaluation of Macular Ganglion Cell Layer Thickness vs Peripapillary Retinal Nerve Fiber Layer Thickness for Glaucoma Detection Using Spectral-domain Optical Coherence Tomography in a Tertiary Philippine Hospital
}

\author{
Luis G Aquino ${ }^{1}$, Norman M Aquino ${ }^{2}$
}

\begin{abstract}
Aim and objective: To appraise the validity of measuring macular ganglion cell layer ( $\mathrm{mGCL}$ ) thickness as an indicator of early glaucoma, as compared to measurement of peripapillary retinal nerve fiber layer (pRNFL) thickness.

Materials and methods: This was a single-center, single-observer, cross-sectional, retrospective study. Records included Filipino adult patients seen from January 2017 onward. Patients underwent testing of both automated visual field (VF) testing with either Humphrey Visual Field Analyzer (24-2 SITA program) or Octopus 311 (G1 program), and standard Spectral-Domain Optical Coherence Tomography (Cirrus HD-OCT 5000). Modified Hodapp-Anderson-Parrish criteria were used to classify subjects as either healthy, suspect, or early glaucomatous eyes. Thickness changes were directly observed through optical coherence tomography. Area under receiver operating curve (AUC) analysis was used to determine ability of $\mathrm{mGCL}$ and PRNFL to discriminate between healthy and early glaucomatous states.

Results: A total of 96 eyes were included. Progressive thinning for all parameters was noted for both pRNFL and mGCL from healthy to suspect to early glaucomatous eyes. The highest AUC of 0.744 was seen in average pRNFL of healthy vs early glaucomatous eyes. However, AUC values for both pRNFL and $\mathrm{mGCL}$ were all above 0.500 .

Conclusion: Measurements of $\mathrm{mGCL}$ thickness in Filipino patients exhibit comparable performance to pRNFL measurements in detecting early anatomic glaucomatous change. It is a tool that can be utilized for early glaucoma detection in addition to current standard diagnostic tests. Clinical significance: This study, the first to be performed on Filipino patients, validates using mGCL thickness as a good parameter in discriminating between normal and early glaucoma patients for this particular population and Ethnic group.

Keywords: Cross-sectional, Ganglion cell complex, Glaucoma, Macula, Optical coherence tomography, Retinal nerve fiber layer.

Journal of Current Glaucoma Practice (2020): 10.5005/jp-journals-10078-1278
\end{abstract}

\section{INTRODUCTION}

Optic neuropathy from glaucomatous changes in the eye is multifactorial in nature and results in irreversible anatomical damage and visual disturbance or loss in patients. Over 111 million individuals worldwide are believed to be affected by glaucoma, making this disease one of the top causes of blindness worldwide. Asia alone accounts for half of those affected. ${ }^{2}$ In the Philippines, it is the third leading cause of blindness among Filipinos, affecting over 70,000 people according to the Third National Survey on Blindness. ${ }^{3}$ However, given the lack of updated and larger-scale studies on its epidemiology, particularly in other third-world countries as well, it is highly possible that these numbers are grossly underestimated. ${ }^{4}$

The hallmark pathology of this disease is progressive and irreversible loss of retinal ganglion cells and their axons. Corresponding changes to a patient's optic disk and visual field (VF) then become manifest as more of these cells become damaged or altered. Change is detected, quantified, and monitored through color, red-free photography, and optical coherence tomography of the peripapillary retinal nerve fiber layer (pRNFL). ${ }^{5,6}$ Standard automated perimetry (SAP) or "White-on-white" VF testing is then used to correlate VF defects to these structural changes. These modalities serve as the current gold standard in glaucoma detection. However, these tests only detect these changes when already $30-50 \%$ of retinal ganglion cells have been irreversibly lost
1,2Department of Ophthalmology and Visual Sciences, Philippine General Hospital, Manila, National Capital Region, Philippines

Corresponding Author: Luis G Aquino, Department of Ophthalmology and Visual Sciences, Philippine General Hospital, Manila, National Capital Region, Philippines, Phone: +632 8554 8400, e-mail: luis. miguel.aquino@gmail.com

How to cite this article: Aquino LG, Aquino NM. Evaluation of Macular Ganglion Cell Layer Thickness vs Peripapillary Retinal Nerve Fiber Layer Thickness for Glaucoma Detection Using Spectral-domain Optical Coherence Tomography in a Tertiary Philippine Hospital. J Curr Glaucoma Pract 2020;14(2):50-56.

Source of support: Philippine General Hospital

Conflict of interest: None

through glaucomatous damage. ${ }^{7}$ Earlier detection of evidence of glaucomatous damage, before irreversible damage has occurred, is therefore ideal. ${ }^{6}$

The macular ganglion cell layer ( $\mathrm{mGCL}$ ) complex has recently been receiving attention as a potential parameter than can be used for early detection of glaucoma. Recent advancements in spectraldomain optical coherence tomography (SD-OCT) technology has allowed the ganglion cell complex (GCC) to be better identified and measured. Multiple studies have investigated the capability of 
this machine to detect changes earlier than the current standard tests for detecting glaucoma, showing that there is significant anatomic change detected in the $\mathrm{mGCL}$, preceding PRNFL and functional changes. This shows that the macular GCC is therefore an earlier indicator of glaucomatous damage. ${ }^{8}$ Oli and Joshi ${ }^{9}$ and Zhang et al. ${ }^{10}$ noted that macular GCC thickness had good ability to detect between healthy and glaucomatous eyes, with area under receiver operating curve (AUC) values of $0.835(p<0.0001)$ and $0.775(p=0.015)$, respectively. Moreno et al. ${ }^{11}$ showed that macular GCC thickness analysis had a more significant role in early glaucoma than that of $\mathrm{PRNFL}$ thickness analysis with AUC values at $0.815(p>0.03)$ compared to $0.735(p<0.03)$, respectively. In terms of progression of the disease, the study by Hirooka et al. ${ }^{5}$ on the use of optical coherence tomography to estimate ganglion cell loss has also been shown to be more accurate during early glaucomatous stages vs advanced cases.

Macular ganglion cell layer changes as a marker for early glaucoma detection presents a novel addition to current diagnostic standards in managing glaucoma. Sound, well-established pathophysiological evidence supports its validity, implying possible profound changes to how we approach glaucoma. ${ }^{12,13}$ Studies have demonstrated its significance in different populations. But current research is still limited. Testing those findings on more populations or geographic areas, such as in a Filipino population, would further support the use of this diagnostic test. Optical coherence tomography analysis of the $\mathrm{mGCL}$ complex can be a possible alternative, or compliment, to PRNFL analysis for early detection of glaucoma. Earlier identification of glaucoma in a patient would have profound implications on the course of the disease.

This study aims to appraise the validity of measuring $\mathrm{mGCL}$ thickness via SD-OCT as an indicator of early glaucomatous disease in the Filipino population. The $\mathrm{mGCL}$ thickness in normal adult Filipino patients in a tertiary eye center will be compared with those suspected of having glaucoma, and those diagnosed with early glaucoma, in order to establish a relationship or trend between mGCL thickness in healthy from glaucomatous eyes. The ability of mGCL thickness measurements vs pRNFL thickness to discriminate the presence of glaucomatous damage will then be determined.

\section{Materials and Methods}

This study is a single-center, single-observer, cross-sectional, retrospective study. Institutional Review Board approval for adherence to the tenets of the Declaration of Helsinki were obtained from the University of the Philippines Manila Research Ethics Board for this study. Identities and general information of subjects included in this study were kept confidential and known only to the principal investigator.

Investigation took place at the outpatient clinic of a tertiary public Philippine hospital. A total of 500 records of patients seen at the Glaucoma clinic from January 2017 to January 2019 were retrieved. Inclusion criteria included Filipino adult patients above 45 years old, who were able to undergo both optical coherence tomography and automated VF testing. One hundred and sixtyfour patients with reliable and acceptable perimetry and optical coherence tomography results were included. Optical coherence tomography results were included if signal strength was above 6/10, while VF results were reliable if the following were met:

- Octopus G1 program with $<15 \%$ reliability factor and $<20 \%$ false-positive and false-negative response rates.
- Humphrey Visual Field Analyzer 24-2 SITA program with $<20 \%$ fixation losses, false-positive and false-negative response rates.

The Hodapp-Anderson-Parrish criteria for diagnosing acquired glaucomatous damage were used to determine the severity of VF damage. Mild or early damage was defined as: $:^{14}$

- Mean deviation (MD) no worse than $6 \mathrm{~dB}$.

- Less than $25 \%$ of points depressed below the $5 \%$ level and $15 \%$ of points depressed below the $1 \%$ level.

- No point within $5^{\circ}$ of the central VF with sensitivity of $<15 \mathrm{~dB}$.

Exclusion from the study was due to the presence of any other ocular comorbidities or congenital pathology, error of refraction greater than +6.00 D myopia or hyperopia, the presence of active ocular inflammation, intraocular surgery within 6 months prior, and intake of systemic medications that would affect the retina or optic nerve. The presence of any factor which might also affect the reliability of the tests to be undertaken excluded a patient from the study, such as presence of media opacities, poor optical coherence tomography signal strength, and poor performance during automated VF testing. A total of 96 charts or eyes were included in the final sample population as shown in Flowchart 1.

Patients eligible for this study were those who already underwent testing via Humphrey Visual Field Analyzer 24-2 SITA program (Carl Zeiss Meditec, Dublin, California) or Octopus 311 G1 (Haag-Streit, Switzerland) program. Data collected from these tests were used as the main data source for the investigation, as well as for stratifying the subjects into each test groups. Part of the criteria per group would include the results of the VF analysis.

Clinical examination performed and as noted in their records was used to aid in stratification, as listed in Table 1.

Changes in thickness of the $\mathrm{mGCL}$ complex and peripapillary retinal layer, measured in micrometers, were directly observed through the diagnostic tests performed on each eye. Macular GCC layer thickness was measured using Cirrus HD-OCT 5000 (Carl Zeiss Meditec, Dublin, California) macular cube $512 \times 128$ protocol with ganglion cell analysis. This protocol measured average, minimum,

Flowchart 1: Flow diagram of selection and inclusion of charts for review

Patient records from 2017 onward of the glaucoma clinic retrieved $(n=504)$

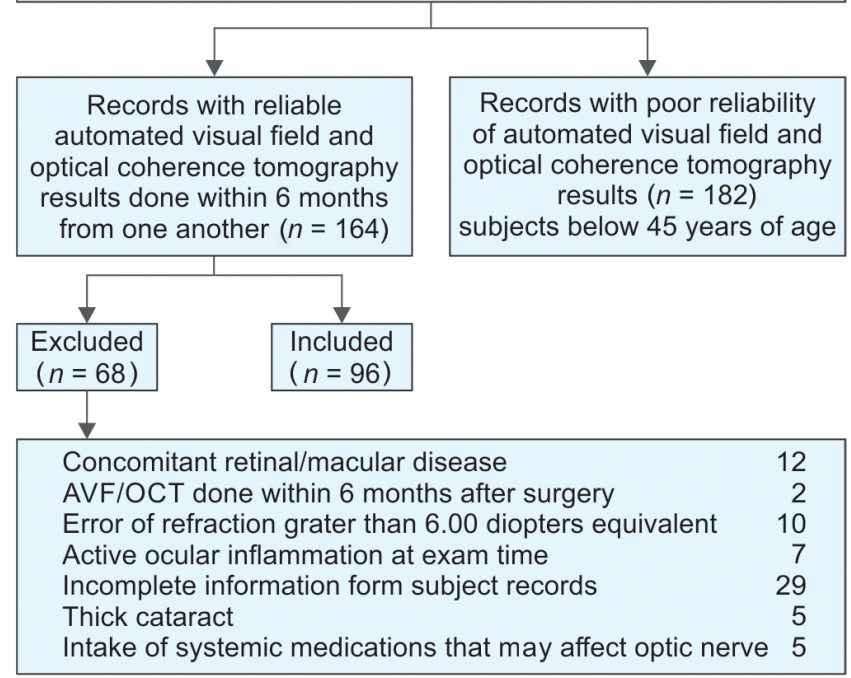


sectoral (superonasal, superior, superotemporal, inferonasal, inferior, inferotemporal) thickness given in micrometers. Peripapillary retinal nerve fiber layer was measured using the same machine using the optic disk cube $500 \times 500$ protocol, giving measurements of average, quadrant, and sectoral (12 clock hours) thickness also in micrometers. Trends or relationships between thickness and the glaucomatous status of the eye were then inferred or correlated from these data.

Statistical computer software specifically Statistical Package for Social Sciences (SPSS) and Matrix Laboratory (MATLAB) were used for statistical analysis in this study. $p$ values $<0.05$ were considered statistically significant. Baseline demographic characteristics of the three groups in this study were obtained and compared for significant difference among groups using post hoc and $t$-test analysis. Thickness measurements of both $\mathrm{mGCL}$ and pRNFL were

Table 1: Clinical parameters to be evaluated for stratification into study groups

\begin{tabular}{|c|c|c|c|}
\hline & Healthy & Suspect & Early \\
\hline $\begin{array}{l}\text { Cup-to-disk } \\
\text { ratio }\end{array}$ & $<0.4$ & $>0.4$ & $>0.7$ \\
\hline ISNT rule & Followed & Not followed & Not followed \\
\hline $\begin{array}{l}\text { Intraocular } \\
\text { pressure }\end{array}$ & $<22 \mathrm{~mm} \mathrm{Hg}$ & $>22 \mathrm{~mm} \mathrm{Hg}$ & $>22 \mathrm{~mm} \mathrm{Hg}$ \\
\hline $\begin{array}{l}\text { Glaucoma risk } \\
\text { factors }\end{array}$ & - & \pm & \pm \\
\hline Automated VF & $\begin{array}{l}\text { Normal } \\
\text { central VF }\end{array}$ & $\begin{array}{l}\text { Normal central } \\
\text { VF or abnormal } \\
\text { central VF in } \\
\text { the presence } \\
\text { of normal disk } \\
\text { findings clinically }\end{array}$ & $\begin{array}{l}\text { Abnormal } \\
\text { central VF }\end{array}$ \\
\hline
\end{tabular}

ISNT, Inferior superior nasal temporal rule; VF, Visual field tabulated and compared for significant difference via one-way analysis of variance (ANOVA). Area under receiver operating curve (ROC or AUC) analysis was then used to compare the findings of mGCL thickness changes vs PRNFL changes. This test measures the ability of a test to discriminate a disease from a normal state. ${ }^{15}$ It also includes the sensitivity and specificity of a given test in the analysis. Hence, the ability of the mGCL complex and the PRNFL to discriminate a healthy from a preperimetric eye to a glaucomatous eye was determined for this particular study population.

\section{Results}

A total of 96 eyes were included in this study: 29 healthy eyes, 37 suspect glaucomatous eyes, and 30 eyes with early glaucoma. Table 2 shows the baseline demographics for each group. Post $h o c$ and $t$-test analyzes were performed to check for significant difference between characteristics per group. Differences in average cup-to-disk ratio (CDR) between each group was statistically significant, while mean VF deviation difference was statistically significant between healthy and early glaucomatous groups and suspect vs early glaucomatous groups. No statistically significant difference based on $p$ value was noted for age, refractive error, and intraocular pressure at examination.

Peripapillary retinal nerve fiber layer mean thickness measurement parameters are presented in Table 3. Mean thickness was generally noted to decrease from healthy to suspect to early glaucomatous eyes. The greatest number of parameters with statistically significant differences was seen in healthy vs early glaucomatous eyes, with eight parameters: Average, inferior, superior, and 1, 6, 7, 11, 12 o'clock mean thickness.

Only one statistically significant difference was identified in mean thickness measurements of $\mathrm{mGCL}$ complex parameters: Average mean thickness between healthy and early glaucomatous eyes. However, as seen in Table 4, there was still a consistent decrease in mean thickness noted for all parameters from healthy

Table 2: Demographic characteristics of the study groups

\begin{tabular}{|c|c|c|c|c|c|c|}
\hline & \multirow[b]{2}{*}{ Healthy $(n=29)$} & \multirow[b]{2}{*}{ Suspect $(n=37)$} & \multirow[b]{2}{*}{ Early $(n=30)$} & \multicolumn{3}{|c|}{$p$ value } \\
\hline & & & & $H v s S$ & $H v s E$ & $S$ vs $E$ \\
\hline Age & $61.551 \pm 8.12$ & $60.514 \pm 12.14$ & $63.700 \pm 8.77$ & 0.909 & 0.691 & 0.404 \\
\hline \multicolumn{7}{|l|}{ Gender } \\
\hline Male & 13 & 15 & 18 & & & \\
\hline Female & 16 & 22 & 12 & & & \\
\hline \multicolumn{7}{|l|}{ Eye } \\
\hline OD & 16 & 17 & 14 & & & \\
\hline OS & 13 & 20 & 16 & & & \\
\hline \multicolumn{7}{|l|}{ Refractive error } \\
\hline \multicolumn{7}{|l|}{ Myopic } \\
\hline Plano & $1.203 \pm 0.83$ & $0.750 \pm 0$ & $1.542 \pm 1.33$ & $0.6222^{\wedge}$ & $0.5458 \wedge$ & $0.5890^{\wedge}$ \\
\hline Hyperopic & 0 & 0 & 0 & & & \\
\hline \multirow[t]{2}{*}{ Intraocular pressure } & $2.000 \pm 1.13$ & $1.648 \pm 0.91$ & $2.107 \pm 1.43$ & 0.529 & 0.951 & 0.341 \\
\hline & $15.448 \pm 6.40$ & $16.270 \pm 6.50$ & $13.900 \pm 3.62$ & 0.832 & 0.555 & 0.216 \\
\hline \multicolumn{7}{|l|}{ Average CDR } \\
\hline \multirow[t]{2}{*}{ Mean VF deviation (dB) } & $0.375 \pm 0.06$ & $0.572 \pm 0.10$ & $0.650 \pm 0.13$ & $<0.001^{*}$ & $<0.001^{*}$ & $0.005^{*}$ \\
\hline & $2.301 \pm 1.77$ & $2.402 \pm 1.70$ & $3.672 \pm 1.55$ & 0.968 & $0.006^{*}$ & $0.008^{*}$ \\
\hline
\end{tabular}

*Post hoc statistically significant difference ( $p$ value $<0.05$ ); $\wedge p$ values calculated using $t$-test

$H$, healthy eyes; $S$, suspect eyes; $E$, early glaucomatous eyes 
Evaluation of mGCL vs pRNFL for Glaucoma Detection Using SD-OCT

Table 3: Comparison of peripapillary retinal nerve fiber layer thickness among the three groups using one-way ANOVA test

\begin{tabular}{|c|c|c|c|c|c|c|}
\hline & \multicolumn{3}{|c|}{$p R N F L$} & \multicolumn{3}{|c|}{ pvalue } \\
\hline & Healthy $(H)$ & Suspect (S) & Early (E) & Hvs S & $H v s E$ & $S v s E$ \\
\hline Average & $98.586 \pm 17.52$ & $90.054 \pm 12.26$ & $84.833 \pm 14.69$ & 0.057 & $0.002^{*}$ & 0.325 \\
\hline Inferior & $126.345 \pm 20.38$ & $118.270 \pm 19.17$ & $101.967 \pm 23.20$ & 0.268 & $<0.001^{*}$ & $0.006^{*}$ \\
\hline Superior & $120.931 \pm 15.42$ & $112.243 \pm 19.59$ & $99.767 \pm 19.37$ & 0.142 & $<0.001^{*}$ & $0.019 *$ \\
\hline Nasal & $70.586 \pm 9.72$ & $70.432 \pm 8.18$ & $66.533 \pm 8.99$ & 0.997 & 0.194 & 0.182 \\
\hline Temporal & $67.345 \pm 14.20$ & $62.243 \pm 13.05$ & $65.867 \pm 14.24$ & 0.299 & 0.911 & 0.535 \\
\hline 1 & $117.655 \pm 22.97$ & $109.000 \pm 22.81$ & $101.133 \pm 26.34$ & 0.318 & $0.026^{*}$ & 0.381 \\
\hline 2 & $84.828 \pm 15.54$ & $78.378 \pm 16.63$ & $77.500 \pm 13.03$ & 0.209 & 0.161 & 0.971 \\
\hline 3 & $60.276 \pm 13.44$ & $56.000 \pm 11.62$ & $57.700 \pm 10.04$ & 0.311 & 0.678 & 0.826 \\
\hline 4 & $69.448 \pm 1.84$ & $62.486 \pm 10.55$ & $65.400 \pm 12.85$ & $0.041^{*}$ & 0.364 & 0.554 \\
\hline 5 & $120.931 \pm 29.93$ & $112.351 \pm 28.08$ & $105.467 \pm 32.63$ & 0.487 & 0.125 & 0.622 \\
\hline 6 & $139.172 \pm 26.59$ & $129.946 \pm 26.84$ & $106.400 \pm 35.54$ & 0.427 & $<0.001^{*}$ & $0.005^{*}$ \\
\hline 7 & $118.759 \pm 32.94$ & $112.703 \pm 26.04$ & $93.767 \pm 25.90$ & 0.664 & $0.003^{*}$ & $0.021^{*}$ \\
\hline 8 & $63.207 \pm 18.25$ & $66.162 \pm 9.92$ & $61.733 \pm 13.19$ & 0.668 & 0.913 & 0.399 \\
\hline 9 & $56.828 \pm 10.78$ & $57.676 \pm 13.54$ & $58.167 \pm 14.77$ & 0.964 & 0.920 & 0.987 \\
\hline 10 & $80.379 \pm 19.07$ & $81.405 \pm 20.81$ & $77.400 \pm 17.01$ & 0.975 & 0.822 & 0.673 \\
\hline 11 & $121.966 \pm 24.05$ & $116.784 \pm 29.51$ & $102.333 \pm 27.46$ & 0.725 & $0.019^{*}$ & 0.085 \\
\hline 12 & $119.793 \pm 29.63$ & $112.405 \pm 22.77$ & $92.600 \pm 27.37$ & 0.500 & $<0.001^{*}$ & $0.008^{*}$ \\
\hline
\end{tabular}

*Significant difference between group $(p<0.05)$

Data are expressed as means in micrometers \pm SDs

$E$, early glaucomatous eyes; $H$, healthy eyes; $S$, suspect eyes

Table 4: Comparison of Macular Ganglion Cell Layer thickness among the three groups using one-way ANOVA test

\begin{tabular}{|c|c|c|c|c|c|c|}
\hline \multirow[b]{2}{*}{ Mean } & \multicolumn{3}{|c|}{$m G C L$} & \multicolumn{3}{|c|}{$p$ value } \\
\hline & Healthy & Suspect & Early & $H$ vs $S$ & $H v s E$ & Svs $E$ \\
\hline Average & $79.621 \pm 8.74$ & $75.812 \pm 9.54$ & $73.667 \pm 10.30$ & 0.248 & $0.049^{*}$ & 0.633 \\
\hline Minimum & $72.966 \pm 12.09$ & $70.838 \pm 13.11$ & $66.500 \pm 15.66$ & 0.806 & 0.170 & 0.404 \\
\hline Superior & $78.138 \pm 8.14$ & $77.027 \pm 10.68$ & $73.233 \pm 13.01$ & 0.910 & 0.195 & 0.331 \\
\hline Superotemporal & $77.379 \pm 18.22$ & $74.649 \pm 12.42$ & $74.233 \pm 10.86$ & 0.713 & 0.665 & 0.992 \\
\hline Superonasal & $80.034 \pm 10.11$ & $76.946 \pm 20.58$ & $77.433 \pm 15.03$ & 0.726 & 0.813 & 0.992 \\
\hline Inferior & $74.655 \pm 9.48$ & $71.568 \pm 11.70$ & $72.100 \pm 10.11$ & 0.470 & 0.625 & 0.977 \\
\hline Inferotemporal & $79.862 \pm 12.17$ & $74.811 \pm 12.83$ & $74.300 \pm 9.94$ & 0.201 & 0.172 & 0.983 \\
\hline Inferonasal & $76.931 \pm 11.17$ & $75.946 \pm 9.86$ & $73.600 \pm 12.06$ & 0.930 & 0.477 & 0.661 \\
\hline
\end{tabular}

*Significant difference between group $(p<0.05)$

Data are expressed as means in micrometers \pm SDs

$E$, early glaucomatous eyes; $H$, healthy eyes; $\mathrm{S}$, suspect eyes

Table 5: Area under receiver operating curve values for average pRNFL and $\mathrm{mGCL}$ measurements

\begin{tabular}{|c|c|c|c|c|c|}
\hline & & Healthy vs suspect & Healthy vs early & Suspect vs early & $\begin{array}{l}\text { Healthy and } \\
\text { suspect vs early }\end{array}$ \\
\hline \multirow[t]{2}{*}{ Average pRNFL } & AUC & 0.643 & 0.744 & 0.655 & 0.694 \\
\hline & $\begin{array}{l}95 \% \text { confidence } \\
\text { interval }\end{array}$ & $0.506-0.780$ & $0.611-0.876$ & $0.514-0.795$ & $0.571-0.817$ \\
\hline \multirow[t]{2}{*}{ Average $\mathrm{mGCL}$} & AUC & 0.590 & 0.668 & 0.572 & 0.614 \\
\hline & $\begin{array}{l}95 \% \text { confidence } \\
\text { interval }\end{array}$ & $0.452-0.729$ & $0.529-0.807$ & $0.432-0.712$ & $0.489-0.739$ \\
\hline
\end{tabular}

to suspect to early glaucomatous eyes for $\mathrm{mGCL}$ thickness measurements.

The area under receiver operating curve (AUC or ROC) values comparing average pRNFL and average $\mathrm{mGCL}$ between groups are presented in Table 5. All AUC values were above 0.500 for both average pRNFL and $\mathrm{mGCL}$ for all group comparisons. AUC for average pRNFL was consistently higher for all comparisons compared to AUC for average $\mathrm{mGCL}$, with the highest AUC of 0.744 seen in healthy vs early glaucomatous eyes. This trend was the same for average $\mathrm{mGCL}$. And AUC value for average $\mathrm{mGCL}$ was also highest in the healthy vs early glaucomatous eyes comparison at 0.668 . 
The AUC curves of average pRNFL and $\mathrm{mGCL}$ were compared between healthy vs suspect (Fig. 1), healthy vs early glaucomatous (Fig. 2), suspect vs early glaucomatous (Fig. 3), and combined healthy and suspect vs early glaucomatous (Fig. 4). These curves show that while AUC value for average $\mathrm{MGCL}$ was consistently lower than average pRNFL. Some areas of its curve were above or equal to the curve for AUC of average pRNFL.

\section{Discussion}

The GCC refers to the three retinal layers involved in early glaucomatous disease process. It consists of the inner plexiform layer (IPL), ganglion cell layer (GCL), and retinal nerve fiber layer

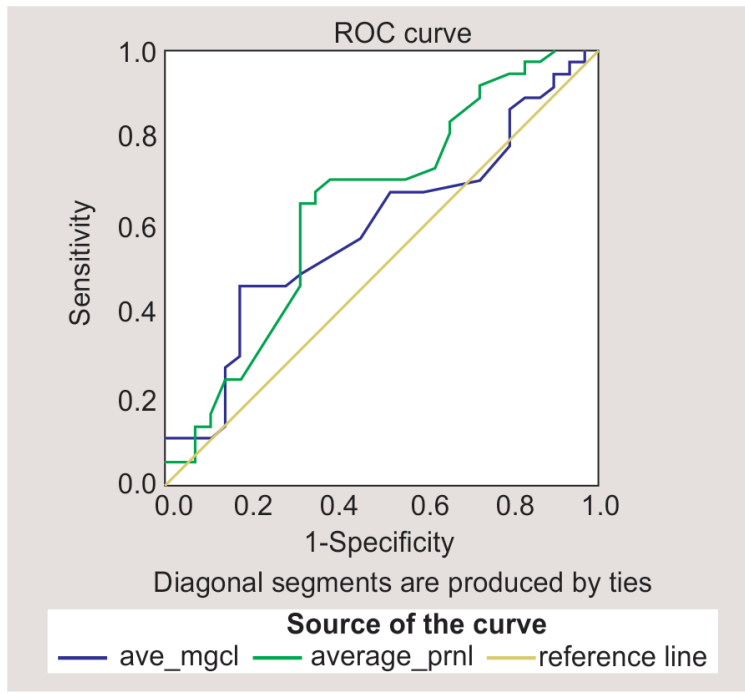

Fig. 1: Comparison of AUC curves for healthy vs suspect average pRNFL and $\mathrm{mGCL}$

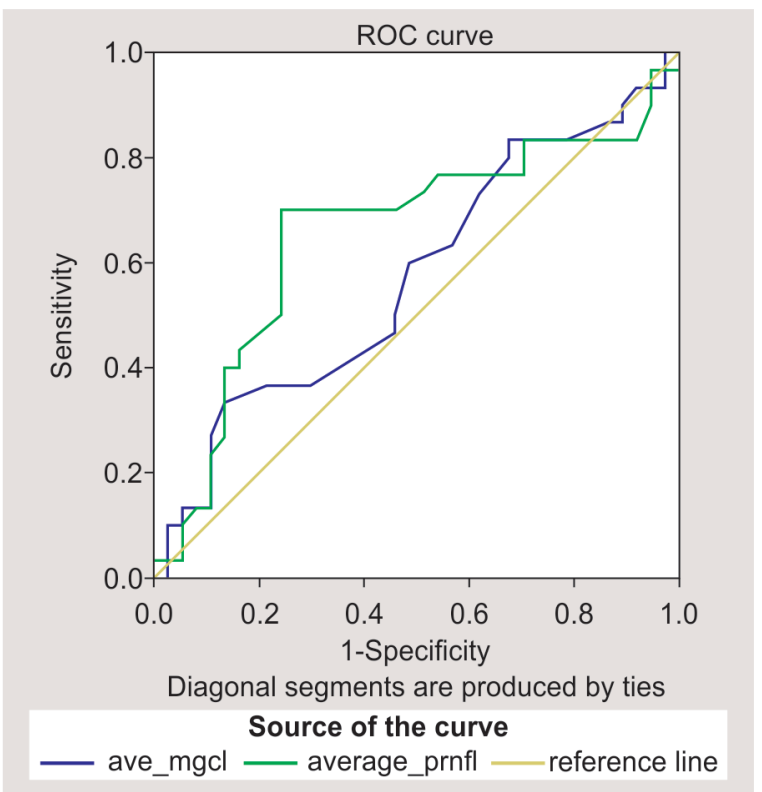

Fig. 3: Comparison of AUC curves for suspect vs early glaucomatous average $\mathrm{PRNFL}$ and $\mathrm{mGCL}$
(RNFL), corresponding to the dendrites, body, and axons of the ganglion cell. This complex is concentrated within the central 5 $\mathrm{mm}$ of the macula and comprises around a third of its thickness. In early glaucomatous damage, a specific sequence of cell death occurs, which starts with the dendritic arbor, then the cell body, then the axon. ${ }^{16}$ Inducible glaucoma models in mice have shown that during acute elevations of intraocular pressure in early stages, pruning of the dendritic arbor occurs, starting off this sequence. Physiological response of the dendrites to light, which is dependent on the morphological differences of this in different patients causes this. ${ }^{17}$ Cell death occurs acutely at this layer causing thinning, before RNFL damage has occurred extensively in other areas. ${ }^{18}$ This implies that ganglion cell death can be detected early, as seen by anatomic

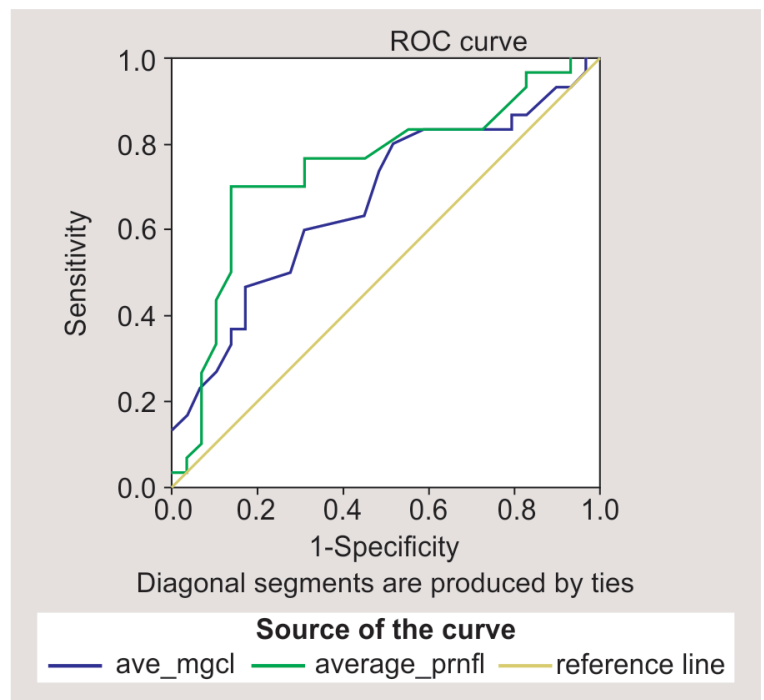

Fig. 2: Comparison of AUC curves for healthy vs early glaucomatous average $\mathrm{PRNFL}$ and $\mathrm{mGCL}$

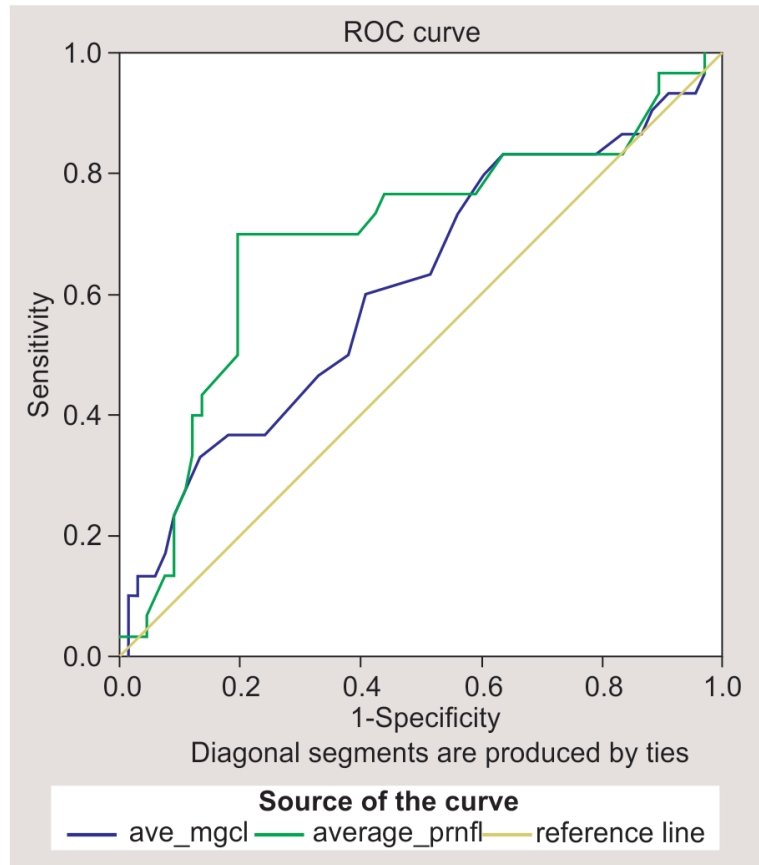

Fig. 4: Comparison of AUC curves for healthy and suspect vs early glaucomatous average $\mathrm{pRNFL}$ and $\mathrm{mGCL}$ 
changes in the macular area. Compared to the circumpapillary RNFL, which receives fibers from nasal and temporal areas, macular GCC contains elements purely from this area alone. Such anatomy gives the area theoretically superior correlation to glaucomatous VF patterns. ${ }^{19}$

The results of this study show that $\mathrm{mGCL}$ thickness measurements of early glaucomatous eyes were thinner as compared to both healthy and suspect eyes as seen in Table 4, thereby supporting this accepted theory of early macular damage. Measurements of pRNFL thickness shown in Table 3 also show progressive thinning from healthy to early glaucomatous eyes, as the axons of the ganglion cells eventually traverse this area as they enter into the optic nerve. Both pRNFL and $\mathrm{mGCL}$ can be therefore considered together, as markers of early glaucomatous damage. ${ }^{18}$

This potential in discriminating healthy eyes from early glaucomatous eyes was better demonstrated in the area under receiver operating curve (AUC) results presented in Table 5. As previously mentioned, this parameter evaluates the diagnostic ability of a test to discriminate a diseased from a normal state. Higher AUC values closer to 1.0 indicate better discriminatory ability. For all group comparisons, average pRNFL and $\mathrm{mGCL}$ both showed good discriminatory ability to differentiate healthy from both suspect and early glaucomatous eyes, with AUC values consistently $>0.500$. The higher AUC values of 0.668 and 0.744 for both $\mathrm{mGCL}$ and pRNFL, respectively, when comparing healthy vs early glaucomatous eyes, show that there is better discriminatory ability when identifying healthy eyes vs eyes with early glaucoma. These show the potential of using optical coherence tomography (OCT)-derived thickness measurements for both regions as early screening tools or adjuncts to clinical parameters and SAP.

Despite the good performance of $\mathrm{mGCL}$ measurements in demonstrating progressive thinning correlated with the severity of early glaucoma, pRNFL values determined by this study showed more statistical significance and higher overall AUC values. Previous studies have also shown pRNFL measurements to perform slightly better than $\mathrm{mGCL}$ in detecting early glaucoma with the use of optical coherence tomography. This was particularly true using the older Stratus time domain-based OCT machines wherein there was limited resolution in dissecting individual retinal layers. ${ }^{13}$ However, even with the use of newer SD-OCT machines such as that used in this study, previous literature also reported higher discriminatory ability of pRNFL measurement vs mGCL. Results obtained through the years by different studies, particularly by Rao et al., ${ }^{20} \mathrm{Na}$ et al., ${ }^{21}$ Yang et al., ${ }^{22}$ and Abdelkader ${ }^{12}$ all showed pRNFL AUC to be higher than mGCL thickness. However, their reported AUC values for $\mathrm{mGCL}$ measurements were still statistically significant in demonstrating good accuracy of the test. Also, of note in one study, that by Kim et al., ${ }^{23}$ is that analysis of the $\mathrm{mGCL}$, while still showing good discrimination, is not a statistically significant parameter for detecting between healthy and glaucomatous eyes, but is still nonetheless above 0.500 in the AUC curve. The results of this study and that study by Kim et al. still show promise for $\mathrm{mGCL}$ to be utilized as an early marker for identifying glaucomatous eyes, especially when pure clinical examination is equivocal.

There was good baseline demographic similarity between analysis groups in this study. Only average CDR was statistically significant between the three groups, while mean VF deviation was statistically significant between healthy and suspect vs early glaucomatous groups. These were expected since the presence of both glaucomatous VF defects and glaucomatous optic neuropathy, seen as enlarged CDR, are part of the clinical diagnosis of glaucoma. The similarity with regards to the other clinical factors between groups shows that baseline demographics were consistent for all analysis groups and non-contributory to the thickness measurements of both the pRNFL and mGCL.

However, the strict inclusion and exclusion criteria for subjects used in this study can mean that the results obtained, while specific and applicable to the study population, is still limited in its applicability to a more widespread population. The study also aimed to validate the use of macular ganglion cell analysis only for early glaucoma in the Filipino population and as such, subjects with advanced glaucoma or other comorbidities had to be excluded from analysis. These criteria therefore limited the study population included in the final data analysis, despite a large number of subjects initially enrolled into the study. Given this, further studies could be undertaken using a larger population of patients to better demonstrate the discriminatory ability of $\mathrm{mGCL}$ and pRNFL thickness measurements for early glaucoma detection, as well as assess its viability as a tool for monitoring progression. Longitudinal studies of these patients could also be performed to confirm present findings.

There is also a lack of a wide normative database for pRNFL and mGCL thickness measurements across different populations. ${ }^{20,21}$ Larger population studies of not just early glaucomatous eyes but also of healthy eyes, could aid in further strengthening our diagnostic threshold and baseline criteria for the significance of $\mathrm{mGCL}$ thickness in early glaucoma. This current study had a limited population as it also excluded the presence of other common retinal diseases and macular pathologies which could influence the thickness of the $\mathrm{mGCL}$. Studying the roles of these diseases together with glaucoma in macular damage could make application of this protocol reach or target a more general population. With further understanding of ganglion cell anatomy and distribution in the macula, studies on sectoral damage correlating to specific VF patterns could expand our knowledge.

\section{CONCLUSION}

This study highlights the potential of $\mathrm{mGCL}$ and pRNFL thickness measurements via OCT as an early screening diagnostic test. Early detection of glaucoma has previously been limited to clinical examination of both structural and functional damage to the optic nerve. With the advancements in optical coherence tomography, examination of the $\mathrm{mGCL}$ has been shown by numerous studies to be a reliable marker for early glaucomatous change.

\section{Clinical Significance}

This study is the first to embark on such involving a Filipino population. Despite limitations, this investigation demonstrated the good potential for this objective evaluation tool in detecting early glaucomatous change.

\section{ACKNowledgments}

Institutional Review Board approval was obtained prior to starting this investigation. Lastly, the authors would like to thank Dr Patricia Marie G Aquino for her assistance with statistical analysis and for the Ophthalmological support staff of our institution. 


\section{Manufacturer Name}

Carl Zeiss Meditec, Dublin, CA: Cirrus HD-OCT 5000 and Humphrey

Visual Field Analyzer

Haag-Streit, Switzerland: Octopus 311

\section{References}

1. Glaucoma, American Academy of Ophthalmology Basic and Clinical Science Course, Volume 10. 2018-2019.

2. Aung T, Crowston J. Epidemiology of glaucoma in Asia. Asia Pacific Glaucoma Guidelines. 3rd ed., Amsterdam, the Netherlands: Kugler Publications; 2016. pp. 2-3.

3. FlorCruz NV, Joaquin-Quino R, Silva PA, et al. Profile of glaucoma cases seen at a tertiary referral hospital. Philipp J Ophthalmol 2005;30(4):161-165.

4. Tham YC, Li X, Wong TY, et al. Global prevalence of glaucoma and projections of glaucoma burden through 2040: a systematic review and meta-analysis. Ophthalmology 2014;121(11):2081-2090. DOI: 10.1016/j.ophtha.2014.05.013.

5. Hirooka K, Izumbata S, Ukegawa K, et al. Estimating the rate of retinal ganglion cell loss to detect glaucoma progression: an observational study. Medicine 2016;95(30):30. DOI: 10.1097/MD.0000000000004209.

6. Weinreb RN, Khaw PT. Primary open-angle glaucoma. Lancet 2004;363(9422):1711-1720. DOI: 10.1016/S0140-6736(04)16257-0.

7. Quigley HA, Katz J, Derick RJ, et al. An evaluation of optic disc and nerve fiber layer examinations in monitoring progression of early glaucoma damage. Ophthalmol 1992;99(1):19-28. DOI: 10.1016/ s0161-6420(92)32018-4.

8. Weinreb RN, Friedman DS, Fechtner RD, et al. Risk assessment in the management of patients with ocular hypertension. Am J Ophthalmol 2004;138(3):458-467. DOI: 10.1016/j.ajo.2004.04.054.

9. Oli A, Joshi D. Can ganglion cell complex assessment on cirrus HD OCT aid in detection of early glaucoma? Saudi J Ophthalmol 2015;29(3):201-204. DOI: 10.1016/j.sjopt.2015.02.007.

10. Zhang C, Tatham AJ, Weinreb RN, et al. Relationship between ganglion cell layer thickness and estimated retinal ganglion cell counts in the glaucomatous macula. Ophthalmol 2014;121(12):2371-2379. DOI: 10.1016/j.ophtha.2014.06.047.

11. Moreno PAM, Konno B, Lima VC, et al. Spectral-domain optical coherence tomography for early glaucoma assessment: analysis of macular ganglion cell complex versus peripapillary retinal nerve fiber layer. Can J Ophthalmol 2011;46(6):543-547. DOI: 10.1016/ j.jcjo.2011.09.006.

12. Abdelkader M. Functional and anatomic assessment of retinal ganglion cells in glaucoma. Delta J Ophthalmol 2016;17(2):65-72. DOI: 10.4103/1110-9173.189076.

13. Tan OU, Chopra V, Lu AT, et al. Detection of macular ganglion cell loss in early glaucoma by fourier domain optical coherence tomography. Ophthalmol 2009;116(12):2305-2314. DOI: 10.1016/ j.ophtha.2009.05.025.

14. Sussana R, Vessani R. Staging glaucoma patient: why and how? Open Ophthalmol J 2009;3(1):59-64. DOI: 10.2174/1874364100903010059.

15. Metz CE. Basic principles of ROC analysis. Semin Nucl Med 1978;8(4):283-298. DOI: 10.1016/s0001-2998(78)80014-2.

16. Lakkis $\mathrm{G}$. The ganglion cell complex and glaucoma. Pharma 2014. 28-32.

17. Risner ML, Pasini S, Cooper ML, et al. Axogenic mechanism enhances retinal ganglion cell excitability during early progression in glaucoma. 2018. ProcNatl Acad Sci USA 115(10):E2393-E2402. DOI: 10.1073/ pnas. 1714888115.

18. Choi JA, Shin H, Park HL, et al. The pattern of retinal nerve fiber layer and macular ganglion cell-inner plexiform layer thickness changes in glaucoma. J Ophthalmol 2017;2017:1-8. DOI: 10.1155/2017/6078365.

19. Salazar JJ, Ramirez Al, De Hoz R, et al. Anatomy of the Human Optic Nerve: Structure and Function. 2018. Intechopen.com.

20. Rao HL, Babu JG, Addepalli UK, et al. Retinal nerve fiber layer and macular inner retinal measurements by spectral domain optical coherence tomography in Indian eyes with early glaucoma. Eye 2012;26(1):133-139. DOI: 10.1038/eye.2011.277.

21. Na JHN, Lee K, Lee JR, et al. Detection of macular ganglion cell loss in preperimetric glaucoma patients with localized retinal nerve fibre defects by spectral-domain optical coherence tomography. Clin and Experimental Ophthal 2013;41(9):870-880. DOI: 10.1111/ceo.12142.

22. Yang Z, Tatham AJ, Weinreb RN, et al. Diagnostic ability of macular ganglion cell inner plexiform layer measurements in glaucoma using swept source and spectral domain optical coherence tomography. PLoS ONE 2015;10(5):e0125957. DOI: 10.1371/journal.pone.0125957.

23. Kim YJ, Kang MH, Cho HY, et al. Comparative study of macular ganglion cell complex thickness measured by spectral-domain optical coherence tomography in healthy eyes, eyes with preperimetric glaucoma, and eyes with early glaucoma. Jpn J Ophthalmol 2014;58(3):244-251. DOI: 10.1007/s10384-014-0315-7. 